CHISAKO YAMAMOTO

Shonan University of Medical Sciences, Japan

\title{
LIFESTYLE AND DIETARY FACTORS ASSOCIATING WITH DEMENTIA STATUS IN THE COMMUNITY-DWELLING ELDERLY AGED 65 YEARS AND OLDER IN A SUBURBAN TOWN OF TOKYO
}

\begin{abstract}
:
Objectives: Dementia is a priority health issue worldwide and becoming the most expensive disease of the 21 st century. The World Health Organization has unanimously adopted a global plan of action on the public health response to dementia 2017-2025 at the 70th World Health Assembly in May 2017. One of the targets included in the plan is risk reduction. An unhealthy lifestyle and diet are likely to cause many diseases. This study aims to clarify lifestyle and dietary factors associating with dementia status in the community-dwelling elderly aged 65 years and older in a suburban town of Tokyo and to clarify gender differences in health behavior. Methods: Self-administered questionnaires were mailed to 2,069 elderly people in February 2004 and 1,538 were returned by addressees or proxies (response rate $74.3 \%$ ). Institutionalized ones were excluded. Analysis subjects were comprised of 52 people with dementia (PWD), 173 people with probable dementia (PPD) and 1,211 cognitively intact people (CIP). Average age(sd) was 74.03(6.55) in men and 75.56(7.27) in women. Descriptive statistics, a chi-square, Kruskal-Wallis, Mann-Whitney $U$ and Bonferroni's multiple comparison tests were performed in men and women, respectively. Significance was set at 0.05 (0.0167 after Bonferroni correction). Lifestyle items included daytime lying hours, sleeping hours, walking/exercise, outing, pet caring, hobbies, smoking, alcohol consumption, cooking and breakfast. Diet items included meat/poultry, soy products, eggs, oily fish, dairy products, fruits, (cooked) vegetables, fried food and miso soup/soup. Results: The Kruskal-Wallis and Mann-Whitney U tests revealed significant differences in most lifestyle items except smoking in men and women. Breakfast was significant only in women. As for diet items, only fruits and fried food were significant in men: CIP>PPD and/or PWD. Women showed significant differences in every diet items: mostly $\mathrm{CIP}>\mathrm{PPD}$ and $\mathrm{CIP}>\mathrm{PWD}$ and only fruits showed PPD $>$ PWD. Conclusion: Though significant differences in lifestyle were weaker in men, CIP showed healthier lifestyle. In women CIP showed better health behavior both in lifestyle and diet. Exercise, social and mental activities like outing and hobbies, moderate alcohol consumption and food items above are recommended to prevent or delay the onset of dementia by Alzheimer's Society. This study, along with Japanese women's better lifestyle, has the same implications associating with dementia risk reduction. It also suggests that Japanese diet "Washoku" (a UNESCO Intangible Cultural Heritage) which is nutritionally well-balanced contributes to better health status as Japanese women enjoy the longest longevity in the world.
\end{abstract}

\section{Keywords:}

dementia, the community-dwelling elderly aged 65 years and older, lifestyle, diet, factors associating with dementia status, risk reduction, health behavior.

JEL Classification: 118 